\title{
G'Libras: Um Sistema Especialista para Auxilio à Criação de Glosas
}

\author{
Cristiane Ellwanger, Cristina Paludo Santos, Pedro Soares, Tiago L. Weisheimer
}

Universidade Regional Integrada do Alto Uruguai e das Missões (URI)

\author{
98.802-470 - Santo Ângelo - RS - Brasil \\ cristianeellwangeregmail.com, paludoesan.uri.br, \\ temqcsoares@gmail.com, tiago.tlw@hotmail.com
}

\begin{abstract}
The morphosyntactic structures and the formation of words, individual or context necessary for automatic Libras translation to Brazilian Portuguese, pose challenges to the development of technologies to deaf community. From this, this article presents G'LIBRAS - an expert system with specific focus on generating glosses, given its importance for the design of accessible texts to the deaf user. The modeling, implementation and system validation was attended by experts (interpreters) and demonstrate efforts towards developing technologies that respect the cultural diversity of the user, their characteristics and their values.
\end{abstract}

Resumo. As estruturas morfossintáticas e a formação de palavras, individuais ou em contexto, necessárias à tradução automática de Libras para a Língua Portuguesa Brasileira, impõem desafios ao desenvolvimento de tecnologias direcionadas a comunidade surda. Diante disso, este artigo apresenta G'LIBRAS - um sistema especialista com foco específico na geração de glosas, dada sua importância para a concepção de textos acessíveis ao usuário surdo. A modelagem, implementação e validação do sistema contou com a participação de especialistas (interpretes) e demonstram os esforços para com desenvolvimento de tecnologias que respeitem a diversidade cultural do usuário, suas características e seus valores.

\section{Introdução}

A tradução da Língua Portuguesa Brasileira para Libras não é um processo direto visto que ambas exploram características próprias na constituição das estruturas linguísticas e na sua articulação e percepção, podendo inclusive impor restrições aos mecanismos gramaticais [de Quadros 2013].

A Libras é dotada de uma gramática constituída a partir de elementos que se estruturam a partir de mecanismos morfológicos, sintáticos e semânticos que apresentam especificidades, mas seguem também princípios básicos gerais [Brito 2011]. Estes princípios possibilitam a produção de um número infinito de construções a partir de um número finito de regras que regem o uso adequado das estruturas linguísticas da LIBRAS. Tais regras podem ser utilizadas na produção de uma interlíngua, também chamada de glosa. A glosa pode ser denominada como um sistema de notação que faz uso de diversas regras para geração de um texto que possa ser traduzido para a língua de sinais de forma a manter uma estrutura sintática e semântica adequada [Felipe 2012].

Para que este processo de transformação seja mais eficiente, este trabalho apresenta o G'Libras - um sistema especialista que dá suporte a criação de glosas, dando 
condições para criação de regras customizadas atreladas ao conhecimento e expertise do profissional de Libras. No entanto, a modelagem de um sistema especialista com este propósito não é trivial e a participação de especialistas da área faz-se necessária em sua concepção, visto que o conhecimento de especialistas em Libras é imprescindível para se obter uma tradução com qualidade na transformação do texto para glosa sem que a comunicação seja prejudicada, pois deve-se manter o sentido do texto no processo de tradução. Além disso, mínimos detalhes podem interferir na transformação e transmitir outro sentido, já que a Libras possui vários sinais, expressões faciais e uma série de movimentos que se empregados de forma incorreta podem mudar o sentido do texto e transmitir a informação distorcida ao surdo.

Dentre as contribuições que o sistema proposto busca oferecer, destacam-se como principais a possibilidade de:

- Otimizar o processo de tradução realizado por interpretes, uma vez que o próprio sistema aplica as regras iniciais de transformação.

- Facilitar o aprendizado do surdo visto que o entendimento e compreensão da língua portuguesa brasileira por parte da comunidade surda, tanto na sua forma oral quanto escrita, é prejudicada em decorrência das diferenças linguísticas;

- Dar um passo em busca de uma notação padrão para as línguas de sinais. Uma vez que ainda se conhece pouco sobre a estrutura das línguas de sinais, todo trabalho que busca descrevê-la, pode resultar em uma melhor compreensão das línguas gestuaisvisuais e,

- Favorecer o desenvolvimento de outras aplicações computacionais que demandam a execução de um processo automático de tradução para Libras.

Além disso, a proposta deste trabalho também se relaciona com dois dos Grandes Desafios em pesquisa citados pela Sociedade Brasileira de Computação - SBC [SBC 2015], que são: (a) Desafio 4 - Acesso participativo e universal do cidadão brasileiro ao conhecimento. Na medida em que se obtêm novos conhecimentos sobre o desenvolvimento de softwares inclusivos, contribui-se para o desenvolvimento de sistemas com melhor usabilidade, acessibilidade e comunicabilidade, e também para o acesso universal ao conhecimento, visto que é fato as vantagens providas pelos recursos computacionais ao desenvolvimento sócio- cognitivo dos indivíduos; (b) Desafio 5 Desenvolvimento tecnológico de qualidade. A proposta visa integrar vários conceitos e técnicas que permitam auxiliar o desenvolvimento e adaptação de tecnologias de apoio à modelagem e implementação de sistemas de softwares inclusivos com qualidade.

Sendo assim, pesquisas envolvendo o escopo desta proposta apresentam-se como ferramentas valiosas. Uma descrição mais detalhada da proposta do G'LIBRAS é apresentada nas seções subsequentes, estruturadas da seguinte forma: a seção 2 apresenta os princípios e teorias que norteiam o desenvolvimento do G'Libras; na seção 3 são apresentadas as regras de conhecimento mapeadas e implementadas no sistema; a seção 4 apresenta o protótipo desenvolvido, bem como os testes realizados a partir dos estudos de casos propostos. Por fim, a seção 5 apresenta as considerações finais.

\section{Princípios Norteadores do Sistema G'LIBRAS}

Acessibilidade é uma preocupação crescente em computação. Uma vez que as informações em ambientes computacionais são apresentadas em sua maioria por meios visuais, pode-se ter a falsa impressão de que a acessibilidade para deficientes auditivos 
não é um problema. No entanto, para pessoas que adquirem a surdez antes da alfabetização, materiais escritos são, em geral, menos acessíveis do que se apresentados em línguas de sinais.

Para o deficiente auditivo a língua de sinais é geralmente a primeira língua adquirida, e ler um texto em uma língua escrita é o equivalente a utilizar uma língua estrangeira. Apesar de um surdo poder ser fluente em português assim como o ouvinte pode ser fluente em língua de sinais, a língua oral e escrita comumente não é sua primeira língua. Este fato limita o acesso a informação por parte da comunidade surda.

Neste contexto, inserem-se as contribuições advindas das pesquisas da área de computação que envolvem o desenvolvimento de tecnologias capazes de favorecer uma sociedade participativa em que o conhecimento possa ser compartilhado e acessado por todos. Nessa seção são abordadas questões importantes que foram consideradas no desenvolvimento deste trabalho, destacando alguns aspectos inerentes ao processo de tradução para Libras e ao desenvolvimento de sistemas baseados em conhecimentos. Além disso, são apresentados alguns trabalhos relacionados ao processo de tradução.

\subsection{Processo de Tradução para Libras}

Tradução, no contexto deste trabalho, é considerado como a passagem de um texto de uma língua para outra língua, mais especificamente trata-se da passagem de textos em Língua Portuguesa para a Língua de Sinais, ambas brasileiras. Esta passagem caracteriza-se como um processo complexo com diferentes etapas em que, em cada uma delas, devem ocorrer análises minuciosas de forma a produzir, como produto final, um texto traduzido que possua coerência morfológica, sintática e semântica.

Efetuar a tradução para Libras envolve conhecimentos referentes às regras que regem a estruturação gramatical da língua e, sobretudo, o conhecimento implícito do especialista que incorpora no processo o significado do que está sendo traduzido. Segundo Rosa [Rosa 2005], "é impossível fazer uma tradução que seja integralmente literal e equivalente, considerando que esta atividade não é um ato mecânico ou de automação, mas sim de translocação de palavras que carregam significados combinados". Neste sentido, as contribuições de especialistas no processo de tradução são fundamentais para o desenvolvimento de sistemas sociais.

Devido às particularidades da estrutura gramatical a Libras não pode ser estudada tendo como base a Língua Portuguesa. A ordem dos sinais na construção do enunciado obedece a regras que refletem a forma como o surdo processa suas ideias com base em sua percepção visual-espacial da realidade. Assim, a estruturação de Libras possui regras próprias como, por exemplo, não são usados artigos, preposições e conjunções visto que estes conectivos estão incorporados ao sinal. Alguns exemplos demonstrados por Strobel [Strobel 1998] demonstram a independência sintática do português. Nos exemplos abaixo, "para" não é usado, pois está incorporado ao verbo (exemplo 1), assim como há alguns casos de omissão de verbos na Libras (exemplo 2).

- Exemplo 1: Libras: EU IR CASA / Português: "Eu irei para casa"

- Exemplo 2: Libras: IDADE VOCÊ / Português: "Quantos anos você tem?"

Assim, compreender a gramática de uma língua é apreender as regras de formação e de combinação dos elementos desta língua, fundamental para o desenvolvimento de sistemas de tradução. A Tabela 1 apresenta uma compilação de algumas das regras de 
estruturação de frases e sentenças existentes na Libras. Uma descrição mais detalhada de outras categorias é apresentada em Felipe [Felipe 2005].

Tabela 1. Regras de sintaxe de Libras

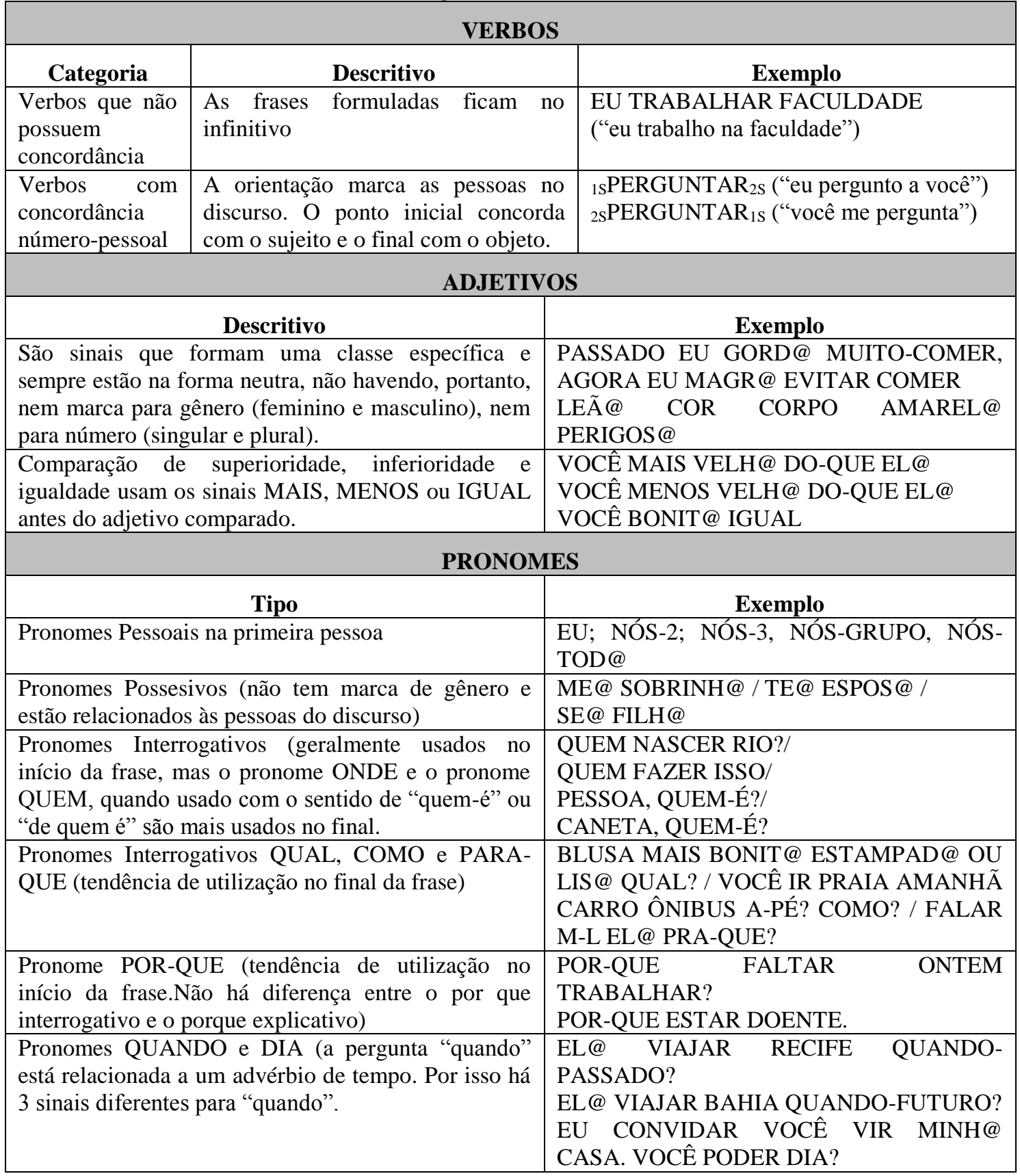

Além das regras gramaticais, a ordem frasal produzida pelo surdo, geralmente, obedece à uma hierarquia semântica, ou seja, os conteúdos de maior significação e importância são colocados em destaque no início da oração. Assim, análises tanto sintáticas, quanto semânticas devem estar envolvidas no processo de geração de glosas com vistas a produção de um texto acessível para o surdo.

\subsection{Sistemas Especialistas e sua aplicabilidade na educação especial}

Muitos projetos se direcionam ao uso de tecnologias na educação de pessoas com necessidades especiais. Entretanto desenvolvedores devem estar cientes da importância 
da aplicação destas tecnologias neste contexto e de como a diversidade influencia os processos de desenvolvimento e a necessidade de se buscar métodos alternativos que realmente favoreçam a inclusão.

Projetos de desenvolvimento de tecnologias direcionados à Educação Especial requerem uma ampla e profunda compreensão do usuário, do seu contexto de uso e da modelagem do ambiente sob os quais são aplicados, que incluí auxílio pedagógico e técnico no que se refere a tecnologias assistivas e adaptativas para sua aplicação [Machado 2012]. Estes conhecimentos ampliam os benefícios provenientes do uso e desenvolvimento de tecnologias e estendem as possibilidades de atuação das ferramentas tecnológicas para o ensino e aprendizagem de pessoas com necessidades especiais

Sistemas Especialistas emergem como ferramentas direcionadas não somente ao armazenamento de conhecimentos, mas também a manutenção e compartilhamento deste. Constituem-se como programas inteligentes que se utilizam de métodos dedutivos para a resolução de problemas técnicos e especializados. Emulam um conhecimento específico, favorecendo à tomada de decisão, a realização de treinamentos para a instrução e servem de subsídio à aplicação de métodos educacionais devido a capacidade que possuem de solucionar problemas que exigem um grau avançado de raciocínio e perícia [Flores 2003].

São constituídos de um motor de inferência que favorece a criação e gerenciamento de regras de forma otimizada, simulando a capacidade humana de tomada de decisão por meio de um raciocínio lógico. Estas regras são sentenças declarativas formuladas a partir de fatos do mundo real associadas ao conhecimento de especialistas humanos de uma determinada área, onde cada uma dessas regras pode ou não estar vinculada a uma condição para ser executada [Coppin 2013]

Este motor de inferência é referenciado como um dos principais elementos que compõe um sistema especialista, sendo responsável pela execução de regras lógicas (de negócio ou de um determinado contexto de aplicação) vinculado a uma base de conhecimento. Assim, sistemas especialistas podem processar regras baseando-se em centenas de milhares de fatos repetidamente de forma confiável, onde o relacionamento complexo de centenas de regras operando em dezenas de fatos concorrentes podem influenciar no resultado de decisões estratégicas importantes.

Estas características os torna bastante utilizados quando se objetiva representar conjuntos de dados para uma determinada área de conhecimento [Coppin 2013]. Características de suma importância dadas as particularidades inerentes a aprendizagem de pessoas surdas e as possibilidades que esta tecnologia proporciona ao reter o conhecimento implícito de especialistas humanos, simular situações reais e propor o aprendizado mais interativo.

\subsection{Trabalhos Relacionados}

A literatura contempla alguns trabalhos relacionados a essa temática, dentre os quais se destacam o SignSim [Campos 2000], o PUL $\varnothing$ [Nunes 2003] e o TLIBRAS [Lira 2014]. O SignSim é uma ferramenta para tradução entre a Libras e a Língua Portuguesa, e viceversa cujo processo de tradução não utiliza nenhum processamento, interpretação ou análise de contexto. Ele volta-se a traduções diretas, baseadas em um dicionário bilíngüe, com a intervenção do usuário em todas as ocorrências de ambiguidade léxicomorfológicas, o que caracteriza a tradução como semi-automática ou assistida. 
O PUL $\varnothing$ trata-se de um sistema de tradução automática unidirecional do português para a representação linear (Libras Script for Translation) da Libras, faz uso da tecnologia de sistemas de tradução automática, auxiliada por humanos, para resolver as ambiguidades/desvios linguísticos e sua entrada de dados constitui-se de um "português normalizado", onde a interlíngua Universal Networking Language - UNL é utilizada para análise semântica do mesmo. Já o TLIBRAS está sendo desenvolvido pelo grupo Acessibilidade Brasil com o objetivo de criar um tradutor automático, em tempo real, do português para a Libras. Neste projeto o processo de tradução automática se dá com base na análise das frases na língua-fonte (português) e posteriormente geração da sentença na língua-alvo (Libras).

A maioria dos trabalhos relacionados utilizam-se de sistemas de tradução constituídos por dicionários de palavras e na verificação da possibilidade de traduzi-las considerando a sua presença ou não em uma base de dados. Com isso, contemplam somente a criação de regras sintáticas no processo de tradução e não direcionam sua atenção aos aspectos formativos da glosa.

Com uma abordagem diferenciada dos sistemas tradicionais, G'LIBRAS oferece condições para criação de regras customizadas, qualificando a glosa e valorizando assim características culturais e regionais da linguagem. Para isso conta com a participação de especialistas humano (intérpretes) que acompanham o processo. Além disso, G'LIBRAS apoia-se na área de Inteligência Artificial e dos subsídios que esta oferece para a concepção de um sistema especialista automatizado que utiliza um framework específico para este propósito. Uma descrição mais detalhada do G'LIBRAS é apresentada a seguir.

\section{G'LIBRAS - Procedimentos Metodológicos}

O G'LIBRAS é um sistema especialista projetado para apoiar o processo de tradução de textos da Língua Portuguesa para a Língua de Sinais, ambas brasileiras. Para sua concepção contou-se com a participação de 5(cincos) especialistas em tradução que auxiliaram na definição das regras que transforma textos em glosas. De acordo com os estudos realizados e as orientações dos especialistas em LIBRAS percebeu-se que não existem modelos de tradução que seguem regras precisas para o processo. Neste caso, as competências e habilidades do tradutor são fatores relevantes para a qualidade da tradução, sendo imprescindível que se tenha domínio sobre as duas línguas para compreender as ideias presentes no texto que estão além das palavras, ou seja, que vão além da gramatica da língua, envolvendo aspectos culturais, sociais e emocionais. A Figura 1 apresenta uma visão geral dos componentes estruturais do sistema.

Dentre as diversas áreas de aprendizado existentes, optou-se pelo uso de fábulas de Esopo da Literatura Infantil para subsidiar os processos de elaboração e avaliação das regras de tradução. Foram criadas glosas para 10 fábulas, no entanto, para fins de exemplificação será utilizada a fábula "Gansa de Ovos de Ouro". Inicialmente foram elaboradas regras padrão que tratam com as diversas especificidades de formação e combinação de elementos, conforme apresentado na Tabela 1. Tais regras envolvem a eliminação/substituição de palavras, letras e preposições, transformação de verbos, tratamento de gênero, dentre outras. Ao todo foram desenvolvidas 85 regras padrão.

Além das regras padrão, outras regras foram elaboradas com a participação direta dos especialistas. Tais regras envolvem as transformações textuais necessárias para refletir corretamente a semântica das estórias e para incorporar as características 
regionais na linguagem. Nesta etapa foram elaboradas, até o momento, 145 regras semânticas envolvendo as especificidades das 10 fábulas consideradas inicialmente.

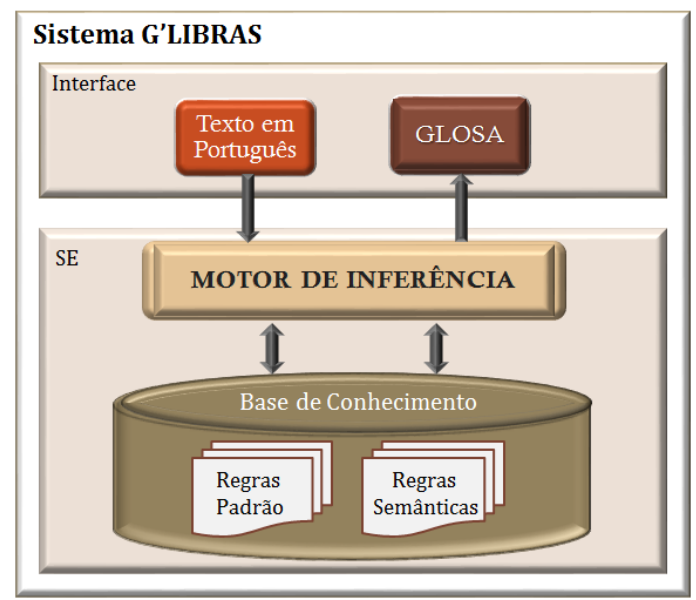

Figura 1. Componentes estruturais do G'LIBRAS

Para a geração da glosa da fábula "Gansa de Ovos de Ouro", por exemplo, foram utilizadas 30 regras semânticas e 39 regras padrão, totalizando a aplicação de 69 regras. A Figura 2 apresenta uma das regras criadas para transformação de verbos para o infinitivo(1a), bem como um exemplo de uma regra semântica utilizada na geração da glosa (1b).

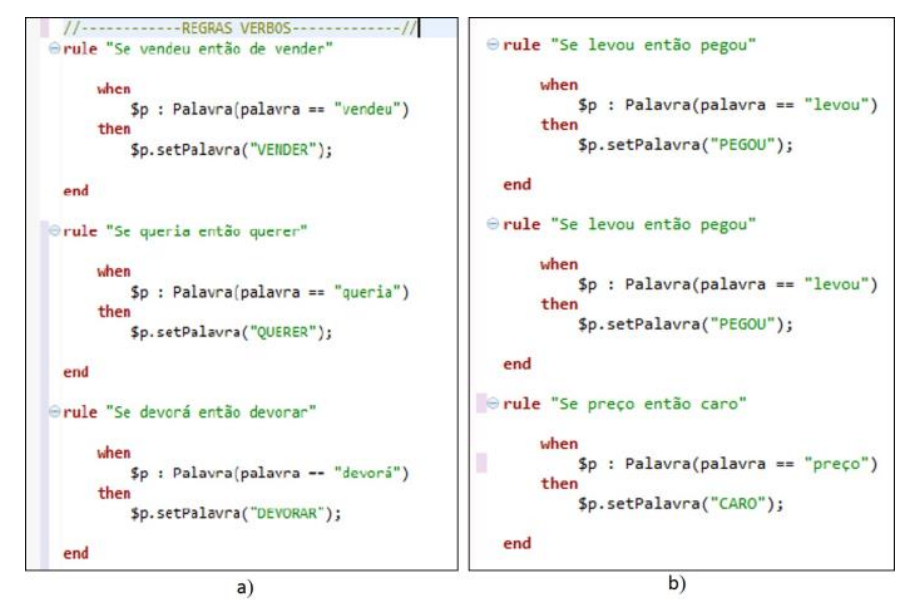

Figura 2. Regras desenvolvidas para geração da glosa.

A quantidade de regras aplicadas na fabula "Gansa de ovos de ouro" reflete a vasta quantidade de conhecimentos inerentes ao processo de geração da glosa e demonstra a viabilidade do uso de sistemas automatizados para este fim. Todas as regras concebidas compõem a base de conhecimento do G'LIBRAS. Para validação das regras utilizou-se a seguinte metodologia: (a) glosas das fábulas foram concebidas manualmente pelos especialistas; (b) um protótipo do G'LIBRAS foi desenvolvido onde glosas foram criadas automaticamente pelo sistema; (c) uma comparação foi realizada entre as glosas da tradução manual e as glosas da tradução automática. Uma descrição mais detalhada do protótipo desenvolvido, bem como dos resultados obtidos é apresentada a seguir. 


\section{Protótipo do Sistema G'LIBRAS}

Para implementação de um protótipo do G'LIBRAS fez-se uso de um framework específico para a implementação de sistemas especialistas referenciado como Drools. Este framework, além de ser open source, permite a criação e gerenciamento de regras e suporta a modelagem de sistemas complexos e com alto grau de conhecimento.

Dentre as principais vantagens desta solução ressalta-se a facilidade de manutenção nas regras, visto que Drools utiliza uma linguagem declarativa em que as regras são descritas em blocos when/then (se/então) simplificando a realização de adaptações ou inclusão de novas regras. Além disso favorece uma padronização na elaboração em termos de desenvolvimento de software proporcionando o seu reuso por outros desenvolvedores. O fluxograma da Figura 3 apresenta a interação e sequência de execução do sistema G'LIBRAS, o qual se estabelece a partir de uma estrutura bottonup formado por três dimensões essenciais.

A primeira dimensão (Drolls) apoia-se nas bibliotecas do framework Drolls para a estruturação de componentes de software necessários para a composição do sistema. Tais componentes envolvem o motor de inferência e a base de conhecimento constituída por conhecimentos iniciais já estabelecidos (memória de fatos), pelo conhecimento implícito de especialistas (memória de regras) e pelo componente "agenda" que referencia a ordem de inserção das regras, bem como a volatilidadedas mesmas, a partir dos quais é realizado o processo de inferência.

A segunda dimensão (sistema especialista) estrutura-se a partir da instanciação do pacote de componentes em que é feita adição de regras. Conforme descrito anteriormente as regras são descritas na linguagem própria do Drools e inicializadas no sistema especialista G'Libras a partir de sua vinculação com o arquivo drl (crédito), o qual permite a agregação de novas palavras.

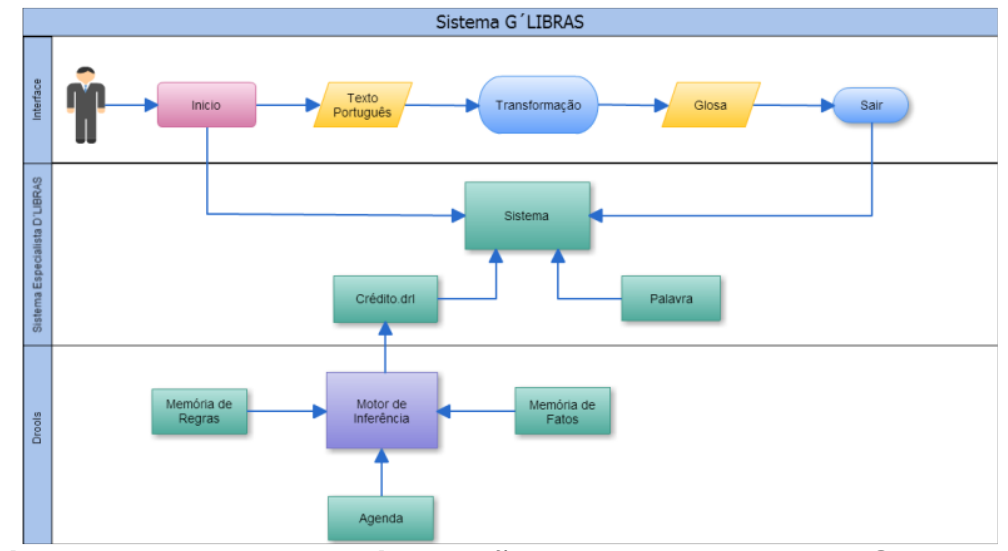

Figura 3. Fluxograma e interações dos componentes G'LIBRAS

A terceira dimensão (interface de usuário) retrata as possibilidades de interação do usuário para com o sistema G'LIBRAS, bem como a criação de glosas. A Figura 4 apresenta a interface proposta neste estágio de implementação do G'LIBRAS, onde gerou-se a glosa proveniente da fábula "Gansa de ovos de ouro", onde foram consideradas as transformações de texto em glosa realizadas por especialistas (Figura 4a) e as transformações realizadas pelo sistema G'LIBRAS. Em comparação às glosas geradas, tanto pelos especialistas quanto pelo G'LIBRAS, verificou-se que o sistema foi mais preciso na tradução, pois agrega em si o conhecimento de 5 especialistas e, associado a forma como as regras foram modeladas e da utilização das funcionalidades 
e otimização no processo de desenvolvimento, proporcionou uma melhor fidelidade da glosa com o texto original.
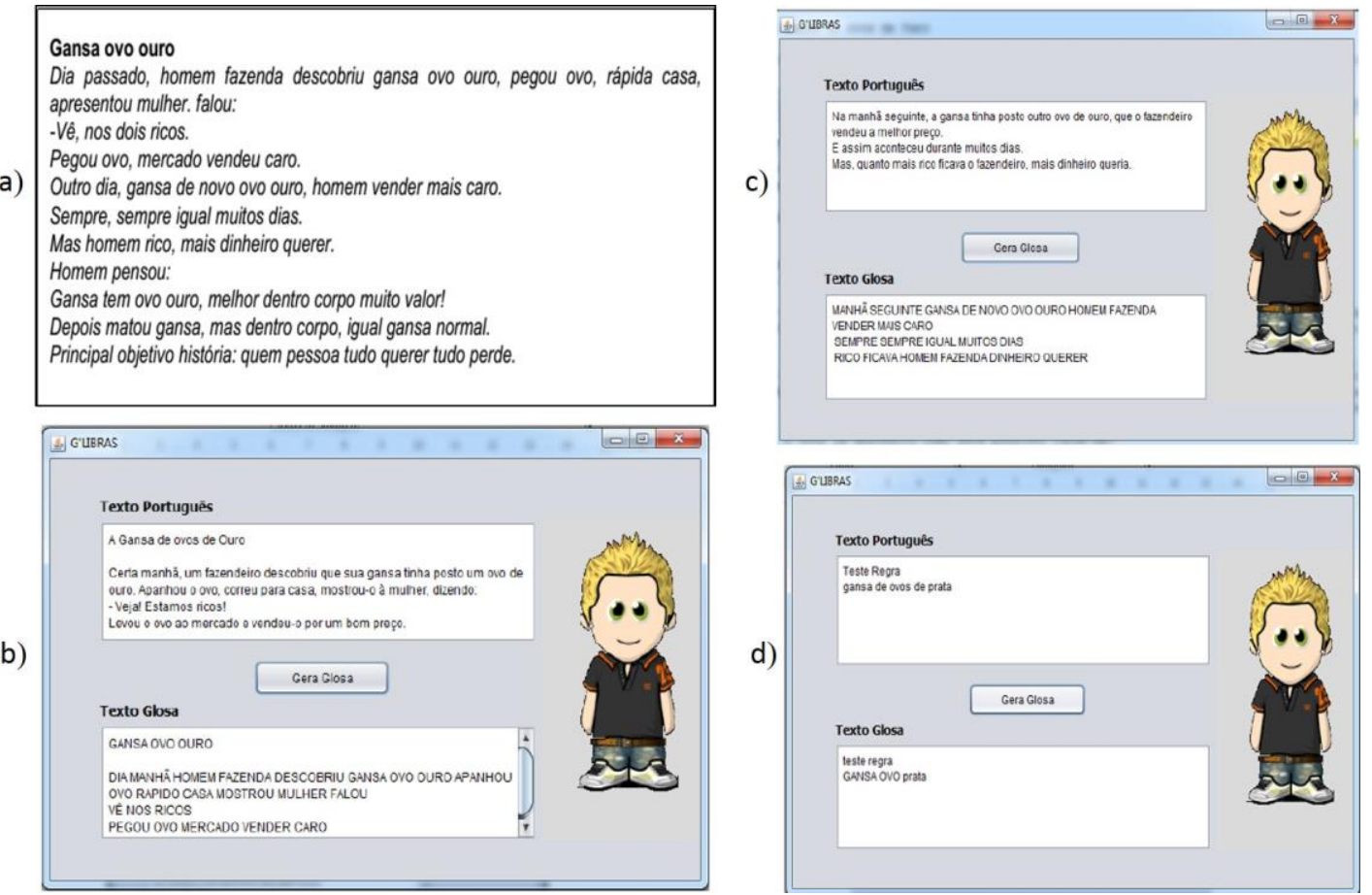

Figura 4. Protótipo do G'LIBRAS

Outra funcionalidade do sistema retratada na interface é a visualização das palavras que não estão na base de regras, representadas com letras minúsculas na glosa gerada (Figura 4d). Isso permite ao especialista visualizar o que deve ser ainda alimentado no sistema via novas regras de conhecimento.

\section{Conclusões}

A ubiquidade, retratada atualmente pelo uso e manipulação de tecnologias de informação e comunicação, estabelece novos desafios a profissionais da computação relacionados com a diversidade e inclusão de pessoas com necessidades especiais. Entretanto, em contextos específicos nem sempre as fronteiras interacionais estão bem definidas, o que exige debates e investigações que busquem soluções alternativas para contemplar aspectos voltados a acessibilidade, permitindo que usuários possam se apoiar em soluções projetadas preservando suas características e especificidades.

Assim, o desenvolvimento de sistemas computacionais para a comunidade surda exige uma série de capacidades e habilidades, não somente dos aspectos peculiares a linguagem de libras e todos os seus pormenores, mas também na seleção e utilização de tecnologias que visem a manutenção e reaproveitamento da estrutura do sistema em termos computacionais que se voltem a estender as aplicações desenvolvidas. Além disso, estratégias devem ser pensadas a fim de envolver o usuário durante o processo de desenvolvimento para que o mesmo possa realmente estabelecer significados aos mesmo. Neste contexto insere-se o presente trabalho, que tem como objetivo a produção de tecnologia respeitando a identidade cultural, suas características e seus valores e considerando uma metacomunicação intercultural no processo de desenvolvimento de modo que este processo realmente estabeleça significado e sentido aos diferentes tipos de usuários que utilizam do sistema. 
Diante disso, cabe destacar que o processo de concepção das regras semânticas contou inicialmente com a participação de 3 especialistas, no entanto, diante de discordâncias dos mesmos em relação a transformação de alguns termos, optou-se por envolver 2 novos especialistas no processo. Assim, as regras elaboradas retratam a opinião da maioria e demonstram que mesmo com a participação de especialistas da área no processo de tradução, nem todos são unanimes quanto à conformidade do produto final, no caso, da glosa gerada.

A partir disso, prevê-se o aperfeiçoamento do sistema com vistas a flexibilizar ao utilizador (interprete) a manipulação e adaptações das regras de tradução conforme suas concepções, a partir da implementação de um módulo adicional ao G'LIBRAS que permitirá a edição das regras a partir de uma interface amigável ao usuário. Dado seu escopo de desenvolvimento, em termos computacionais, esta aplicação foi desenvolvida com vistas a dar suporte a implementação de outras aplicações que se direcionem a auxiliar no processo de aprendizagem dos deficientes auditivos, auxiliando não somente professores na elaboração de material didático para este público, mas também os próprios surdos em na compreensão e entendimento de determinados conteúdos

\section{Referências}

ROSA, Andréa da Silva. Tradução, Fidelidade e Sobrevida. In: Entre a visibilidade da tradução dos sinais e a invisibilidade da tarefa do intérprete. Campinas, SP: Arara Azul, 2005.

STROBEL, K.L.; FERNANDES, S. Aspectos lingüísticos da língua brasileira de sinais. Secretaria de Educação. Superintendência de Educação. Departamento de Educação Especial. Curitiba: SEED/SUED/DEE, 1998.

FELIPE, Tanya A.; MONTEIRO, Myrna. LIBRAS em contexto: Curso Básico: Livro do professor. Brasília, MEC/SEESP n ${ }^{\mathbf{0}}$ Edição, v. 7, 2005.

FELIPE, Tanya A. Bilinguísmo e surdez. Trabalhos em Lingüística Aplicada, v. 14, n. 1, 2012.

CAMPOS, Márcia Borba et. al. SignSim: uma ferramenta para auxílio à aprendizagem da língua brasileira de sinais. In: V Congresso Ibero-Americano de informática na Educação - RIBIE, Chile (2000).

LIRA, G. TLibras Tradutor Digital Portugues x Língua Brasileira de Sinais-Libras.Boletim Técnico Senac, 2003. Disponivel em: http://www.senac.br/BTS/293/boltec293d.htm. Acesso em: Fevereiro/2014.

Nunes, M.G.V., Pelizzoni, J. M., Greghi, J. G., Hasegawa, R., Martins, R. T. Projeto PULO. NILC Project Report, Jun. (2003).

Machado, Vagner Vaz, Uso de Tecnologia na Educação do Surdo na Escola Regular, Faculdades de Tecnologia de São Paulo, 2012.

FLORES, C.D.. Sociedades Artificiais: a nova fronteira da inteligência nas máquinas.2003.

COPPIN, Ben. Inteligência Artificial. Ed. LTC, 2013.

SBC - Sociedade Brasileira de Computação. Grandes Desafios da Pesquisa em Computação no Brasil 2006 - 2016. Relatório, 2006. Disponível em http://www.sbc.org.br/. Acesso: março/2015.

BRITO, Lucinda Ferreira. A Gramática Da Libras. 2011.

de QUADROS, Ronice Muller. Alfabetização e o sentido da língua de sinais. TEXTURA: ULBRA, v. 2, n. 3, 2013. 